\title{
Neutron lifetime experiment with pulsed cold neutrons at J-PARC
}

\section{Go Ichikawa, ${ }^{a, b, *}$ Yasuhiro Fuwa,${ }^{c}$ Takuro Hasegawa, ${ }^{d}$ Masahiro Hino, ${ }^{e}$ Katsuya Hirota, ${ }^{a}$ Takashi Ino, ${ }^{a}$ Yoshihisa Iwashita, ${ }^{e}$ Masaaki Kitaguchi, ${ }^{f}$ Jun Koga, ${ }^{g}$ Shun Matsuzaki, ${ }^{g}$ Kenji Mishima, ${ }^{a, b}$ Takanori Mogi, ${ }^{h}$ Koki Morikawa, ${ }^{d}$ Hiroki Okabe, ${ }^{d}$ Hidetoshi Otono, ${ }^{i}$ Yoshichika Seki, ${ }^{j}$ Daiichiro Sekiba, ${ }^{k}$ Tatsushi Shima, ${ }^{l}$ Haruki Shimizu, ${ }^{m}$ Hirohiko Shimizu, ${ }^{d}$ Naoyuki Sumi, ${ }^{a}$ Hirochika Sumino, ${ }^{n}$ Satoru Yamashita, ${ }^{o}$ Kodai Yano $^{g}$ and Tamaki Yoshioka ${ }^{i}$}

${ }^{a}$ High Energy Accelerator Research Organization (KEK), Tsukuba, 305-0801, Japan

${ }^{b}$ J-PARC Center, Tokai, 319-1195, Japan

c Japan Atomic Energy Agency, Tokai, 319-1195, Japan

${ }^{d}$ Department of Physics, Nagoya University, Nagoya, 464-8602, Japan

${ }^{e}$ Institute for Integrated Radiation and Nuclear Science, Kyoto University, Kumatori, 594-0494, Japan

${ }^{f}$ Kobayashi-Maskawa Institute for the Origin of Particles and the Universe (KMI), Nagoya Unversity, Nagoya, 464-8602, Japan

${ }^{g}$ Department of Physics, Graduate School of Science, Kyushu University, Fukuoka, 819-0395, Japan

${ }^{h}$ Department of Physics, The University of Tokyo, Tokyo 113-0033, Japan

${ }^{i}$ Research Center for Advanced Particle Physics (RCAPP), Kyushu University, Fukuoka, 819-0395, Japan

${ }^{j}$ Center for Physics and Mathematis, Osaka Electro-Communication University, Neyagawa, 572-8530, Japan

${ }^{k}$ Institute of Applied Physics, University of Tsukuba, Tsukuba, 305-8573, Japan

${ }^{l}$ Research Center for Nuclear Physics (RCNP), Osaka University, Ibaraki, 567-0047, Japan

${ }^{m}$ The Graduate University for Advanced Studies, Hayama, 240-0193, Japan

${ }^{n}$ Department of General Systems Studies, Graduate School of Arts and Science, The University of Tokyo, Tokyo, 153-8902, Japan

o International Center for the Elementary Particle Physics (ICEPP), The University of Tokyo, Tokyo, 113-0033, Japan

E-mail: go.ichikawa@kek.jp

A free neutron decays in a lifetime of about $880 \mathrm{~s}$. Although the neutron lifetime is an important parameter in modern physics, there is a $4.6 \sigma(9.5 \mathrm{~s})$ discrepancy between the experimental results of two typical methods: the beam method and the storage method. We are carrying out a new experimental method to measure the neutron lifetime using a pulsed neutron beam at J-PARC MLF BL05 to determine the cause of the discrepancy, whether overlooked systematic uncertainties or new physics. A review of our experiment including the first physics result with acquired data during 2014 - 2016 and the status using new neutron optics are presented here.

*** Particles and Nuclei International Conference - PANIC2021 ***

*** 5 - 10 September, $2021 * * *$

*** Online ***

\footnotetext{
${ }^{*}$ Speaker
} 


\section{Introduction}

A free neutron decays into a proton, an electron, and an antineutrino through the weak interaction in a lifetime of $879.4 \pm 0.6 \mathrm{~s}$ [1]. Since the neutron is one of the simplest baryons made up of the lightest quarks, the lifetime is an important parameter in modern physics, such as predicting ${ }^{4} \mathrm{He}$ abundance by Big Bang Nucleosynthesis and determination of $V_{\mathrm{ud}}$ term in the Cabibbo-KobayashiMaskawa matrix. In recent years, much attention has been paid to the $4.6 \sigma(9.5 \mathrm{~s})$ discrepancy between the experimental results of the neutron lifetime by two typical methods: the beam and the storage methods (Figure 1). The beam method measures the neutron flux and decay protons by different detectors, and the storage method counts survival neutrons after some storage times. The discrepancy is called the neutron lifetime puzzle. Solving the puzzle is an urgent task, though whether the cause is overlooked systematics or new physics is still discussed and not yet settled.

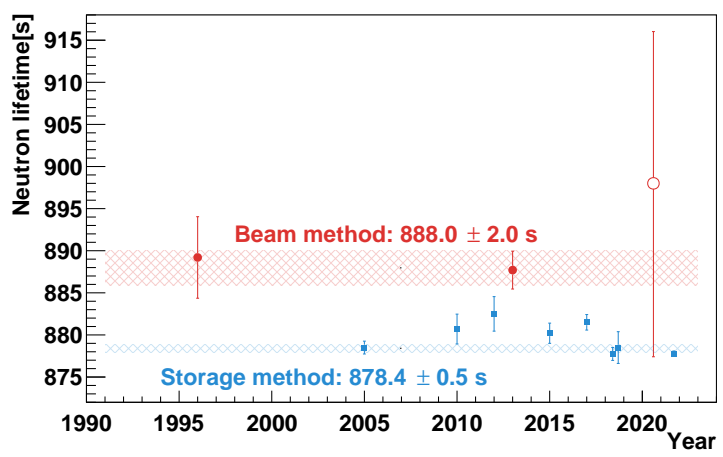

Figure 1: Measured values of the neutron lifetime. Red (blue) points correspond to the results of beam (storage) methods. The means and standard deviations of each method are also shown. The open red circle indicates the first result of our experiment.

\section{The experiment at J-PARC}

We have been carrying out a neutron lifetime measurement with a new method using a pulsed neutron beam at J-PARC MLF BL05 to solve the neutron lifetime puzzle [2]. We measure the neutron flux and decay electrons simultaneously by a Time Projection Chamber (TPC) [3] filled by working gas and ${ }^{3} \mathrm{He}$. The schematic of the apparatus is shown in Figure 2. To reduce the background event rate, the neutron beam is shaped to bunches shorter than the length of the sensitive region by the Spin Flip Chopper (SFC) [4]. Background events induced by (n, $\gamma$ ) reactions of upstream or downstream the TPC can be separated by a time-of-flight (TOF) analysis. Tracks of charged particles are drifted upward to a multi-wire proportional chamber (MWPC), and their waveforms are recorded and analyzed. Since the neutron flux and decay rate are counted by the same detector, the systematic uncertainty is different from the typical beam method. We are aiming for $1 \mathrm{~s}(0.1 \%)$ precision determination of the neutron lifetime to achieve a definitive result.

The neutron lifetime $\tau_{\mathrm{n}}$ is derived in our experiment as

$$
\tau_{\mathrm{n}}=\frac{1}{\rho \sigma_{0} v_{0}}\left(\frac{S_{\mathrm{He}} / \varepsilon_{\mathrm{He}}}{S_{\beta} / \varepsilon_{\beta}}\right),
$$




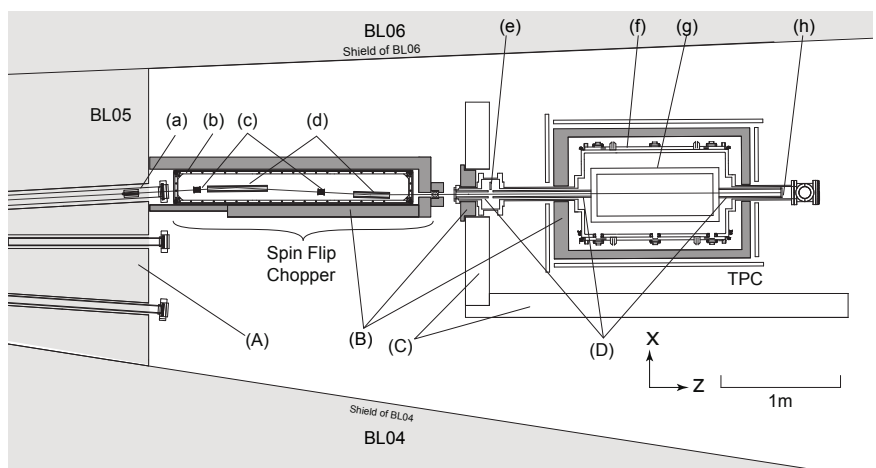

Figure 2: The schematic of apparatus[2]: (A) concrete shield, (B) lead shield, (C) iron shirld, (D) ${ }^{6} \mathrm{LiF}$ beam collimator, (a) short-wavelength pass filter, (b) guide coil, (c) resonance spin flipper coils, (d) magnetic super mirrors, (e) neutron switching shutter, (f) vacuum chamber, (g) TPC, and (h) ${ }^{6} \mathrm{LiF}$ beam catcher.

where $\rho$ is the ${ }^{3} \mathrm{He}$ density in the gas, $\sigma_{0}$ is the cross section of ${ }^{3} \mathrm{He}(\mathrm{n}, \mathrm{p})^{3} \mathrm{H}$ reaction at the neutron velocity of $v_{0}, 5333 \pm 7 \mathrm{barn}, v_{0}$ is the thermal neutron velocity of $2200 \mathrm{~m} / \mathrm{s}, S_{\mathrm{He}}$ and $S_{\beta}$ are the numbers of experimentally observed ${ }^{3} \mathrm{He}(\mathrm{n}, \mathrm{p})^{3} \mathrm{H}$ and $\beta$ decay events respectively, and $\varepsilon_{\mathrm{He}}$ and $\varepsilon_{\beta}$ are the detection efficiencies of each event estimated by a Geant 4 based Monte Carlo simulation.

The first physics result with acquired data during $2014-2016$ was $\tau_{\mathrm{n}}=898 \pm 10_{\text {stat }_{-18}}^{+15}$ sys $\mathrm{s}$, plotted in Figure 1 with other experimental results. Though this result demonstrates the feasibility of our method, uncertainty reduction is needed to solve the neutron lifetime puzzle. The systematic uncertainty was dominated by the neutron bunch correlated background of ${ }_{-14}^{+2} \mathrm{~s}$. Hence, upgrading the neutron optics is necessary to reduce both statistical and systematic uncertainties.

\section{Upgrading of neutron optics}

We upgraded neutron optics before and during the beamtime of J-PARC in the first half of 2021. To make use of the neutron beam, the apertures of neutron optics upstream the TPC were increased as summarized in Table 1. A collimator made of lead and LiF is placed just upstream of the short-wavelength pass filter. The short-wavelength pass filter is made of super mirrors of m-value $m=3$ aligned like wedge shapes. The magnetic super mirrors are m-value of $m=5$ mirrors and components in the SFC. The resonance spin flipper coils are placed in newly designed holders to retain the high spin flipping ratio. The holders block the environmental magnetic field from magnets for polarizing magnetic mirrors and keep the inner uniform field of about $1 \mathrm{mT}$.

\section{Results and outlook}

We carried out experimental runs after the upgrading of neutron optics, including successful physics runs with MLF beam power of $600 \mathrm{~kW}$. The TOF distributions of ${ }^{3} \mathrm{He}(\mathrm{n}, \mathrm{p})^{3} \mathrm{H}$ reaction event candidates of before and after the upgrading are shown in Figure 3. The number of bunches is increased from 5 to 6 , and the total normalized neutron flux was increased by 3.0 times. By this upgrading, we can reach $1 \mathrm{~s}(0.1 \%)$ statistical accuracy within 60 days of beam time. Additionally, the high flux measurement facilitates the systematic study of background events by various event 


\begin{tabular}{l|cc} 
Component & Before upgrading & After upgrading \\
\hline Collimeter & $20 \times 25 \mathrm{~mm}^{2}$ & $30 \times 50 \mathrm{~mm}^{2}$ \\
Short-wavelength pass filter & 4 mirrors of $60 \times 150 \mathrm{~mm}^{2}$ & 6 mirrors of $110 \times 140 \mathrm{~mm}^{2}$ \\
Magnetic super mirrors & $5+5+5$ mirrors of $140 \times 25 \mathrm{~mm}^{2}$ & $8+8+10$ mirrors of $200 \times 100 \mathrm{~mm}^{2}$ \\
Resonance spin flippers & $25 \times 25 \times \pi \mathrm{mm}^{2}$ & $70 \times 50 \mathrm{~mm}^{2}$ \\
\hline Neutron flux inside TPC & $5.7 \times 10^{5} 1 / \mathrm{s}[3]$ & $1.7 \times 10^{6} 1 / \mathrm{s}$
\end{tabular}

Table 1: The increase of apertures of neutron optics and the neutron flux for MLF beam power of $1 \mathrm{MW}$. The magnetic super mirrors are separated to 3 stages and the numbers of mirrors of each stage are indicated.

selections. We measured the phase-space distribution of the neutron beam after the SFC to trace the direct and scattered neutrons. We are now studying to identify the sources of $(n, \gamma)$ backgrounds and going to shield them from neutrons in the next beam time to reduce the systematic uncertainty.

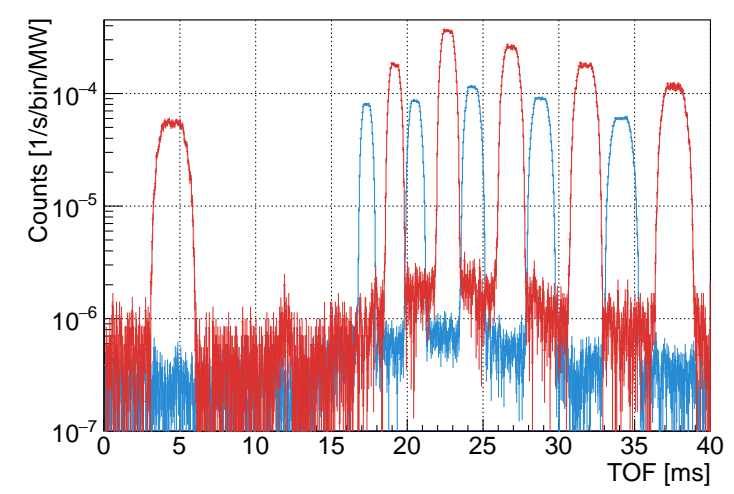

Figure 3: The TOF distributions of ${ }^{3} \mathrm{He}(\mathrm{n}, \mathrm{p})^{3} \mathrm{H}$ reaction event candidates of before (blue) and after (red) the upgrading. The counts are scaled to the MLF beam power of $1 \mathrm{MW}$ and 1 bin corresponds to $0.4 \mathrm{~ms}$.

\section{References}

[1] Particle Data Group, The review of particle physics, Prog. Theor. Exp. Phys. 2020 (2020) $083 \mathrm{C} 01$.

[2] K. Hirota, G. Ichikawa, S. Ieki, T. Ino, Y. Iwashita, M. Kitaguchi et al., Neutron lifetime measurement with pulsed cold neutrons, Prog. Theor. Exp. Phys. 2020 (2020) $123 \mathrm{C} 02$ [2007.11293].

[3] Y. Arimoto, N. Higashi, Y. Igarashi, Y. Iwashita, T. Ino, R. Katayama et al., Development of time projection chamber for precise neutron lifetime measurement using pulsed cold neutron beams, Nucl. Instrum. Meth. Phys. Res. A 799 (2015) 187 [1503.07936].

[4] K. Taketani, T. Ebisawa, M. Hino, K. Hirota, T. Ino, M. Kitaguchi et al., A high S/N ratio spin flip chopper system for a pulsed neutron source, Nucl. Instrum. Meth. Phys. Res. A 634 (2011) S134. 